\title{
The Basic Principles of Instructional Design of TEFL Based on Knowledge Management
}

\author{
Xianzhi Hu \\ Research Center for Applicable Foreign Languages, Yibin University, Yibin 644000, China \\ huxianzhi@188.com
}

\begin{abstract}
This paper focuses on the basic principles of instructional design of TEFL based on knowledge management. It can integrate discipline-specific learning resources to efficiently proceed with actual instructional design based on knowledge management, thus, enhancing personal knowledge accumulation for organization knowledge management, classification and orderly process in accordance with the actual needs of absorbing knowledge, and facilitating creative solutions to the problems in teaching.

Index Terms - knowledge management; instructional design; basic principles; people-oriented; TEFL; knowledge innovation
\end{abstract}

\section{I . Introduction}

Now we are in the information age. The highlight of economic globalization makes English an important communication tools, which has become the most widely used language in all areas of human life. It is an essential issue in the research on the instructional design at present how to encourage learners' study and development in the era of knowledge economy undoubtedly. The knowledge management is a process applied to enterprises for acquiring, storing, studying, participating and innovating in order to enhance the productivity, capability of meeting an emergency and quick-reaction of organizations or individuals successfully. It is already a new starting point in the research on the instructional design field instantly to apply the successful experience of enterprise's knowledge management to the instructional design, and to regard learning and teaching as a course of knowledge collecting, propagating, sharing and producing new knowledge, and then design all steps.

The research considers that in the practical instructional design, the knowledge management, which is capable of integrating knowledge resources of the learning and teaching, and of integrating the knowledge and information of individuals into the knowledge of organizations, and of categorizing, sorting out knowledge according to the actual requirement of learning and teaching, and organizing, storing knowledge based on their relationships, is beneficial to accumulating and sharing knowledge in order to solve problems in the process of learning and teaching. This thesis is tempted to focus on the significance of the knowledge management as well as the comprehensive improvement of the learning and teaching ability through the application of the knowledge management in the practice of the college English instructional design. Therefore, there is a conclusion on the principles of the instructional design. That is "focusing on accumulation with the human-oriented view; mutual trust and sharing development; making innovations of knowledge through constant communication".

\section{II . Knowledge and knowledge management}

A. Knowledge

For a long time, the knowledge is epistemological object of study in philosophy, so our common knowledge definition mostly derives from the philosophical perspective. We can also identify the entity and the nature of all things non-defined knowledge. China's education class dictionaries popular definition of knowledge: "understanding of the properties of things and links, the performance for the perception of things, appearance, concepts, rules, and other psychological form of" [1], or, more specifically: "The so-called knowledge properties and contact a reflection of the objective things, it reflects the objective world and the subjective mapping in the human brain, it reflects the form of activities, sometimes expressed as the perception or appearance of the main things sensual is a perceptual knowledge, and sometimes are rational knowledge concepts or laws about things. " ${ }^{[2]}$ The definition stressed that knowledge is a subjective mapping ${ }^{[3]}$, is based on philosophical epistemology, the theory of reflection of the objective world.

Knowledge from the point of view of human cognition (concept map [4] Figure 2-1 made by using Mindjet Mindmanager) will inevitably involve the explicit knowledge and tacit knowledge.

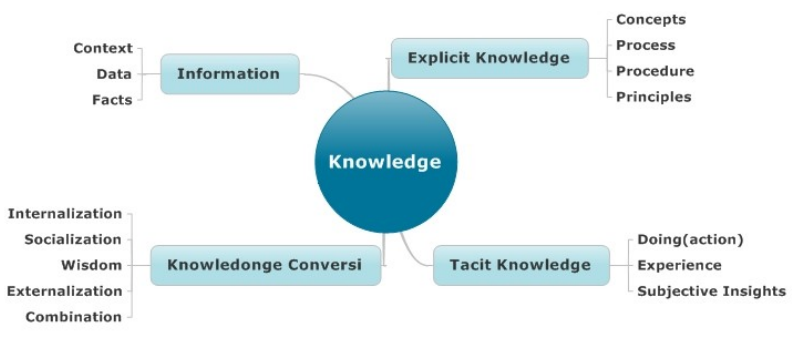

Figure 2-1 Mind Map of Knowledge

It is particularly important for us to understand the concept of tacit knowledge and explicit knowledge and their application and transformation mutually. Furthermore, it is increasingly obvious that the key factor of knowledge management and knowledge innovation is the constant 
accumulation of the individuals' tacit knowledge their transformation into organizational knowledge. ${ }^{[5]}$

Explicit knowledge, just as its name implies, is such kind of knowledge that can be spread through language and words. The typical explicit knowledge mainly refers to the knowledge of the existence form of patents, scientific invention and special technology, the presence of medium with the books, computer databases, CD-ROM, etc.. Tacit knowledge (The Implicit Knowledge), also commonly known as "Tacit Knowledge (Tacit Knowledge), is often an individual or organization has the knowledge accumulated over a long period and is usually difficult words, it is difficult to spread. For example, the highly skilled chefs or artists can reach the world level technology or skills, are very difficult to express language, so as to impart to others or share with others. Division of explicit knowledge and tacit knowledge breakthroughs in the past, people of knowledge, understanding, and recognition without systematic processing experience-based knowledge. If explicit knowledge is "the tip of the iceberg", tacit knowledge is hidden below the surface of the water the majority, although they are more than explicit knowledge is difficult to find, but it is the most important source of social wealth. Knowledge management is an important management tacit knowledge than explicit knowledge better, but also create value, the ability to tap and utilize tacit knowledge is the key to the success of individuals and organizations.

\section{B . Knowledge Managements}

Management aimed at ensuring project/task progress and quality of processes or products involves a collection of activities such as situation assessment, goal definition, team development, resource allocation, and the kind. Knowledge is typically defined in terms of collections of rules, principles and structured information that enable people to make decisions and solve problems. It is the driving force for business and social innovation and progress and produces series of influences for organization to keep up with this advancement of knowledge. It also challenges the academic institution/universities, which often have strong social responsibilities and just experienced transaction from peripheral to main stream. In this knowledge society, the traditional management is not able to meet the demands of the development. Knowledge management thus should be considered as a new model of management for this development. Knowledge management initially belongs to the area of business administration (corporation management), but it is not purely limited to business management only. Knowledge management principle and techniques should also apply to the academic institutions for the organization, distribution of knowledge resources, to better promote the organizational goals of the universities. Knowledge management, then, is a technology(Figure 2-2 drawn by Mindjet Mindmanager) that focuses on the knowledge involved in a set of problem situations or in a system.

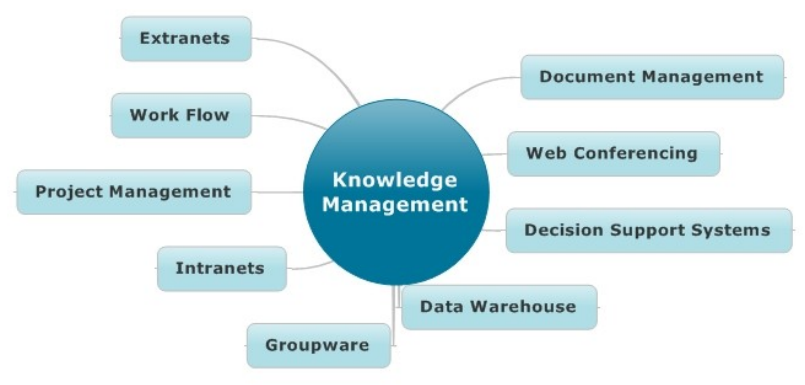

Figure 2-2 Mind Map of Knowledge Management ${ }^{[6]}$

The notion of a system as a collection or combination of disparate, but identifiable, components organized to facilitate accomplishment of goals is essential in order to understand KM. A system is a complex and dynamic collection of different things, including people, information, administrative processes, and the kind. One aspect of management is to make use of the effective and efficient functioning of system components. KM focuses on the knowledge components within a system, some knowledge may be explicitly represented (in the form of information databases, policies and procedures, for example) and some may be implicit (in the form of tacit knowledge, organizational culture, habits, etc.). One goal of KM is to facilitate the transformation of implicit knowledge into accessible explicit knowledge that can be brought to bear in relevant problem-solving situations.

\section{Instructional design}

Instructional design (ID) professionals aim to improve individual and organizational performance. It may be defined as a systematic approach to instructional planning that typically involves a project team analyzing a problem situation, exploring alternative performance support or instructional solutions, and then planning, implementing, evaluating, and managing solutions. This process is often poorly-structured and iterative, and is involved in people from different backgrounds and areas of expertise.

Gagne and Briggs' definition: "The teaching is to promote learning styles affect learners' series of events."

Wu Meina stresses: "Teaching is a human activity with a clear purpose, but teachers teaching and students' learning are unified into activities with fundamental purpose so as to promote students' learning, and to enable students to acquire a certain amount of knowledge and skills for better development mentally and physically"

Pi Liansheng: "Teaching is through the dissemination of information to promote specific learning objectives of the students to achieve the expected activities the purpose of teaching is to enable students to master the knowledge previously did not know, the original does not have the skills to form the original attitude, thus based on the original development of the intelligence of the students." Teaching and Learning mechanism valued its various customers in the process of education and three of such categories are described 
below. A typical model of such system is indicated in Figure 3-1: Generalized teaching-learning process $\operatorname{model}^{[7]}$

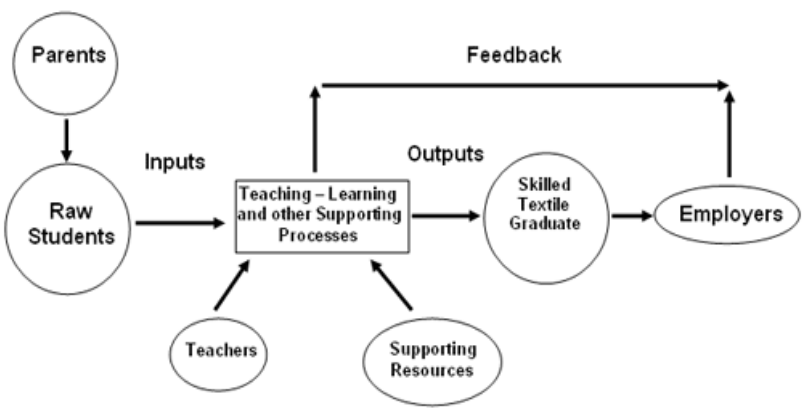

Figure 3-1: Generalized teaching-learning process model ${ }^{[7]}$

The first to propose the concept of instructional design is the famous educational psychologists in the United States the Gagne (Robert Gagne, 1999). His two masterpieces: "The conditions of learning and teaching theory" and "teaching design principles for advancing the science of psychology and education combined and enhance the scientific level of instructional design, at least make the contribution of the following four areas:

1.vague concept made clear explanation. Educational theory and long-term;

2.established a study to learn the new system;

3.learning conditions elaborated on the basis of the proposed new system of a Pedagogy;

4.proposed a set of Principles of Instructional Design and Technology.

Gagne, "The purpose of teaching is to promote learning" ${ }^{[8]}$ according to his book of "The conditions of learning and teaching". In this regard, we can interpret it this way: assume that students learn in a particular environment they will be able to demonstrate the expected behavior. In other words, you need to arrange the external situation, in order to activate, support and maintenance of the internal process constitute a learning event. Based on this thinking, Gagne thought about the concept of "Instructional Design (ID)". Since Gagne put forward the concept of "ID", the domestic and foreign educators have been studying hard to apply the theory of instructional design into practice as well as the development of teachers specialist courses. Scholars all over the world, based on their in-depth discussion of the concept of "instructional design", have summed up roughly the following perspective: ${ }^{[9]}$

1.Instructional design system plan or planning teaching process.

2.The learning experiences and learning environment of Instructional design for the creation and development of technology.

3. The Instructional Design is a science of design.

The following model(Figure 3-2) designed by Dick and Carry is a systematic instructional design model influenced by Gagne's theories of instructional design.

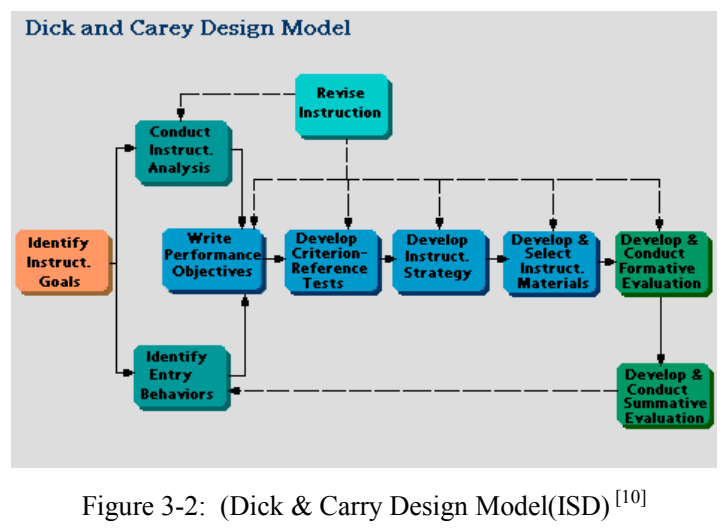

$\mathrm{Wu}$ Meina thinks that instructional design is to solve a series of complex instructional problems so as to find the best solution for the process of the operation which must be carried out by the teachers to master basic skills in instructional design or specialized personnel. "[11]

Because of the epitaxial overlap among instructional design, instructional development, curriculum development and other terms, as well as the complex background of personnel who participate in instructional design, people's understanding to the connotation of instructional design is not entirely the same. Thus, everyone thinks in his way. We believe that, with the deepening of the theory into practice, people's realization of instructional design is essentially bound to continuously improve.

ID professionals are required to resolve complex issues, such as connecting learning and performance objectives with assessable outcomes. Several people are often involved in these problem-solving processes. Some team members may work on one task/aspect of an instructional or performance solution (developing assessment measures, for example) while other team members on a different ID problem (for example, storyboarding specific lessons). ID activities may be accomplished at different times as well as at different locations. In short, ID is a complex, collaborative enterprise, requiring careful planning and management in order for goals to be achieved.

\section{The basic principles of instructional design of TEFL based on knowledge management}

In the process of instructional design practice based on knowledge management the author gradually came to an assumption: If we focused on the accumulation of knowledge from the principles of people-oriented and overcome the obstacles of knowledge sharing on the basis of mutual trust, as well as communicate constantly in order for knowledge innovation, it would be helpful for us to creatively solve the problems in the process of teaching. Nowadays, more and more people are increasingly growing awareness of the importance of knowledge management. If we define that the essence of management is "trust", which represents social capital, then the nature of knowledge management is to achieve a shared innovation based on "people-oriented" mutual trust and continuous exchange. In fact, this is a 
knowledge management-based problem solving process. Therefore, we believe that: "focusing on accumulation with the human-oriented view; mutual trust and sharing development; making innovations of knowledge through constant communication".

Both for the learning organization or learner himself, the accumulation of knowledge is the basis for the implementation of knowledge management. Is a learning organization, since it runs from the first day, there will be a lot of information and knowledge. If there is no accumulation of information and knowledge will disappear with the passage of time or personnel changes. It is the gradual accumulation of information and knowledge, it contributed to the personal and social development, social culture. Therefore, learners and learning organizations must pay attention to the accumulation of information and knowledge. This is the primary condition for knowledge management. Knowledge base, formed by the accumulation of knowledge and information base, but also one of the main objects of knowledge management.

Knowledge management is not only the accumulation of knowledge, sharing of knowledge, the essence of knowledge management is that it can provide the exchange of knowledge space. Knowledge management is to create an environment conducive to learners exchange organizational structure and culture of any learning organization, through some mechanism, so that learners are able to get the full exchange and communication. So as to maximize the information and knowledge exchange process fusion and sublimation, knowledge exchange to be inspired and improve. If the accumulation and sharing of knowledge to solve the problem of explicit knowledge dissemination, knowledge exchange tacit knowledge will be disseminated also varying degrees. It is worth noting that the recessive encoding and exchange them, not just macro planning can be completed, but starting from a specific transaction and a small part of the patiently continuously.

\section{Conclusion}

We believe that knowledge management-based instructional design has the following characteristics:

(1)For the purpose of exchange of knowledge transfer and innovation;

(2)The entire process is focused on learners' knowledge collection, classification, sharing their new knowledge generation, not just passing knowledge of teachers to students, but triggering learners' initiatives and innovation;

(3)It is rather necessary through the entire design process to provide learners with a positive and effective shared interaction environment as well as learners' equality and mutual assistance.

Instructional design based on knowledge management purpose is to more effectively guide the students through the explicit knowledge easily be overlooked in the teaching, but more importantly, the tacit knowledge. Including knowledge structure, learning ability and innovative thinking through the acquisition and creation of knowledge in the learning process, the learner not only on quantitative knowledge has been accumulated and will be greatly enriched and improve.

Moreover, based on knowledge management "peopleoriented emphasis on accumulation; mutual trust, shared to improve; continuous exchange, knowledge innovation" of teaching design philosophy can help us (whether it is teaching designers, subject teachers or students) more rational target management performance assessment better design and understanding of formative assessment and summative evaluation. Much Japan and the United States respected management guru Deming warned very clearly: "The performance appraisal, regardless of call it the control of management or other names, including management by objectives, is the only USA Today management is the most destructive force." [12] into the concept of knowledge management in instructional design, we will be able to maximize the large number of teaching problems to solve in the design phase. We believe that knowledge management ideas, methods will be integrated in the broader field of education!

Be extended space with the content of the traditional classroom-based instructional design knowledge management, providing learners with a wealth of shared resources, is strengthened among teachers, between teachers and students, the exchanges between the life and life to promote learners' personalized learning enhanced sense of participation, stimulate enthusiasm for learning, and really train learners a strong self-learning ability and capacity for sustainable development.

\section{Acknowledgment}

Fund project(11SA105): Key project of Humanities and Social Science of The Educational Department of Sichuan Province, China.

\section{References:}

[1] Gu Mingyuan Education Dictionary, Volume 1, Shanghai Education Press, 1990, 144

[2] Dong Chuncai Encyclopedia of China Education, China Encyclopedia Publishing House, 1985 Edition, 525

[3] Pi Lian Sheng Wang Xiaoming Pang Weiguo Lin Ying Education Psychology Shanghai Education Press, 2004

[4] [5] Pi Liansheng Instructional design - The theory and technology of psychology, Beijing: Higher Education Press, 2000, pp. 28-29

[6] Xianzhi Hu, The Application of Knowledge Management in Instructional Design-Taking the Instructional Design of College English As a Case East China Normal University, 2007

[7] Liu Jie Modern Instructional Design Capital Normal University Press, 2005.1 Page 2

[8] RM Gagne. Conditions of learning and teaching theory. Shanghai: East China Normal University Press, 1999, page 20

[9] Kekang He Instructional System Design Beijing Normal University Press, 2002.10, pp. 2-3

[10] Walter Dick, Lou Carey, James O.Carey The Systematic Design of Instruction, Fifth Edition 2002.10 pp16-17

[11] Wu Meina. Instructional design, Beijing: Higher Education Press, 1994, pp11-13

[12] Dongfei Long, Deming Mmanagement and Enterprise practice Machinery Industry Press, 2004 